Classification

Physics Abstracts

$42.10-42.65-42.70$

\title{
Giant optical non-linearities of critical micro-emulsions (*)
}

\author{
E. Freysz, M. Afifi, A. Ducasse \\ Centre de Physique Moléculaire Optique et Hertzienne, Université de Bordeaux I, \\ 33405 Talence Cedex, France
}

B. Pouligny and J. R. Lalanne

Centre de Recherche Paul Pascal, CNRS, 33405 Talence Cedex, France

(Reçu le 5 novembre 1984, accepté le 14 janvier 1985)

\begin{abstract}
Résumé. - L'autofocalisation d'un faisceau laser continu dans une micro-émulsion a été mise en évidence et analysée. Elle révèle une non-linéarité optique géante de ce matériau non-linéaire original. Les mesures de puissance seuil d'autofocalisation conduisent à des valeurs d'indice non-linéaire $n_{2}$, qui divergent lorsque l'on se rapproche d'un point critique $\left(n_{2} \gtrsim 10^{-8} \mathrm{~cm}^{2} \mathrm{~W}^{-1}\right)$. Cette divergence est directement liée au comportement critique de la compressibilité osmotique du milieu.

Abstract. - Self-focusing of a cw laser beam in a micro-emulsion has been demonstrated and analysed. Giant optical non-linearities of this novel non-linear medium are reported. The non-linear index $n_{2}$ deduced from the threshold power for self-focusing is found to increase divergently when approaching a critical point $\left(n_{2} \gtrsim 10^{-8} \mathrm{~cm}^{2} \mathrm{~W}^{-1}\right)$. This divergence is related to the critical behaviour of the osmotic compressibility of the medium.
\end{abstract}

\section{Introduction.}

Optical materials exhibiting very large non-linearities in a broad fréquency range are required in many applications such as four wave mixing techniques. It has been recently suggested [1] that a suspension of dielectric particles could be a good candidate in this domain. Indeed giant non-linear optical indices of the order of $10^{-9} \mathrm{~cm}^{2} \mathrm{~W}^{-1}$ have been measured in polystyrene latex sphere suspensions by means of self-focusing and phase conjugation $[2,3]$. The non-linear response of the medium is due to the radiation pressure acting on the spatial distribution of the spheres (electro-striction), which results in a spatial modulation of the refractive index of the medium.

This Letter deals with electro-striction in micro-emulsions, a kind of material which had not been previously envisaged in the search of new non-linear optical components. Micro-emulsions are thermodynamically stable mixtures of an oil, water and a surfactant and often a cosurfactant (alcohol) [4]. Such liquid phases are fluid and optically isotropic. It has been shown that diluted micro-emulsions feature a micellar structure. In the case of water in oil micro-emulsions, water is in the form of spherical droplets a few nanometers in diameter, isolated from the oil rich phase

$\left(^{*}\right)$ This work was supported in part by « Direction des Recherches, Etudes et Techniques (Paris) ». 
by a shell of surfactant molecules (inverted micelles) $[4,5]$. The stability of the system is partly controlled by attractive interactions between the micelles. Critical-like behaviours, resulting in a separation between two identical phases, have been reported in the recent years from light scattering experiments [6]. Critical micro-emulsions are characterized by the divergence of a number of quantities, such as the correlation length $\xi$ of the fluctuations of the refractive index and the osmotic compressibility $K_{T}$. In section 2 we show that the divergence of $K_{T}$ results in giant values for the non-linear index $n_{2}$. This prediction is confirmed by the experimental results reported in section 3 , which are based upon the self-focusing of a continuous wave laser beam in a water / sodium dodecyl sulfate (SDS) / butanol / toluene system.

\section{Basic principles.}

The micro-emulsions of interest here are assumed to be constituted with micelles, that we will consider as spherical polarizable particles of refractive index $n_{\mathrm{a}}$ suspended in a host medium of refractive index $n_{\mathrm{b}}$. If a non-uniform electric field is imposed on the system, each particle experiences a force proportional to the field gradient [1] :

$$
F=\frac{1}{2} \alpha \nabla \overline{E^{2}}
$$

where $\overline{E^{2}}$ is the quadratic mean value of the field. $\alpha$ is the effective relative polarizability of the particle and is given by [3] :

$$
\alpha=n_{\mathrm{b}}^{2} \frac{\left(n_{\mathrm{a}} / n_{\mathrm{b}}\right)^{2}-1}{\left(n_{\mathrm{a}} / n_{\mathrm{b}}\right)^{2}+1} a^{3}
$$

where $a$ is the particle radius.

In the case of a water in oil micro-emulsion, such as that used in the experimental section, the mean refractive index of the reverse micelles is lower than that of the continuous phase, which is mainly constituted with oil, so that $\alpha<0$ from equation (2). That means that the micelles are repelled from the regions where the electric field is highest.

The radiation pressure or - equivalently - the increase in the osmotic pressure $\pi$ in the system is given by [1]

$$
p(r)=\delta \pi=\frac{1}{2} \alpha N(r) \overline{E^{2}}
$$

where $N(r)$ is the number density of particles at point $r$.

Assuming that the field varies slowly over a distance of the order of the correlation length $\xi$ of the fluctuations of $N(r)$, the induced variation of the micelle concentration $\delta N(r)$ can be calculated to first order in $\bar{E}^{2}$ from first principles as a simple function of the osmotic compressibility $K_{T}[1]$ :

$$
\delta N(r)=\frac{1}{2} \alpha N^{2}(r) \overline{E^{2}}(r) K_{T}
$$

with

$$
K_{T}=\frac{1}{N}\left(\frac{\delta N}{\delta \pi}\right) .
$$

The local effective index of refraction resulting from equation (4) is a linear function of the field intensity $I(r)$ if $\delta N(r) \ll N(r)$ :

$$
n(r)=n_{0}+n_{2} I(r)
$$


with

$$
I(r)=\frac{c}{4 \pi} n_{0} \overline{E^{2}} \quad(\text { Gaussian units) }
$$

$n_{2}$ is the so-called non-linear index of refraction. From (4) and (6) :

$$
n_{2}=\left(\frac{2 \pi}{n_{\mathrm{a}}}\right)^{2} \frac{1}{c}\left(N_{0} \alpha\right)^{2} K_{T}
$$

where $N_{0}$ is the equilibrium (zero field) number density. If $\phi$ is the volume fraction of the micelles in the medium, then $n_{2}$ is proportional to $\phi a^{3} N_{0} K_{T}$. The solid particle suspensions which have been tested up to now as non-linear media correspond to $\phi \lesssim 10^{-2}$ and $a \gtrsim 10^{-7} \mathrm{~m} \mathrm{[2].} \mathrm{Inter-}$ actions between particles are more or less negligible so that $N_{0} K_{T} \simeq(k T)^{-1}$ (where $k$ and $T$ are respectively the Boltzmann constant and the temperature). The micro-emulsions investigated in this work correspond to $\phi \sim 10^{-1}$ and $a \sim 10^{-8} \mathrm{~m}$. The smallness of the micellar radius compared to that of solid particles is obviously not favourable for obtaining large non-linearities. However, attractive interactions between micelles are known to give rise to critical points [6] which, at first sight, can be thought of as analogues to the liquid-gas critical point. At the critical point, both $K_{T}$ and $\xi$ go to infinity. The pretransitional behaviour of those two quantities is described by the usual scaling laws :

and

$$
\begin{gathered}
K_{T} \sim\left(\frac{T_{\mathrm{c}}-T}{T_{\mathrm{c}}}\right)^{-\gamma} \\
\xi \sim\left(\frac{T_{\mathrm{c}}-T}{T_{\mathrm{c}}}\right)^{-\nu}
\end{gathered}
$$

Here the critical point is assumed to be approached by varying the temperature $T . T_{\mathrm{c}}$ is the critical temperature. The system investigated in the experimental section features a lower critical point, e.g. the single phase domain corresponds to $T<T_{\mathrm{c}}$. The values of the critical exponents should be 1.24 and 0.63 if the analogy with the liquid gas case were complete. However, definitely different values have been found for a number of micellar systems [7]. The non-linear index $n_{2}$ diverges according to equation (9). Therefore non-linearities even larger than those measured for solid particle suspensions should be observed in the close vicinity of a micro-emulsion critical point. At the same time the divergence of the correlation length (see Eq. (10)) should lead to a critical slowing down of the relaxation of the spatial distribution of the micelles toward equilibrium.

The simplest experiment allowing the measurement of $n_{2}$ is the self-focusing of a Gaussian laser beam in the non-linear medium $[8,9]$. The non-uniform transverse intensity profile of the beam imposes an inhomogeneous distribution of $n(r)$ (Eq. (6)). Since the value of $n_{2}$ given by equation (8) is positive whatever the sign of $\alpha, n(r)$ is maximum on the beam axis and the medium behaves as a non-linear convergent lens, the focal length of which decreases when the power $P$ of the beam is increased. In the absence of saturation processes of the non-linearity [9], and of laser-induced heating of the material, the self-focusing of the beam occurs as soon as $P$ is larger than a threshold power given by [10]

$$
P_{T}=\frac{0.146 \lambda_{0}^{2}}{n_{0}} \frac{1}{n_{2}}
$$

where $\lambda_{0}$ is the laser wavelength. Equation (12) is a straightforward relation between $P_{T}$ and $K_{T}$. The threshold power is expected to decrease critically when $T \rightarrow T_{\mathrm{c}}$. 


\section{Experimental results.}

The experimental set-up used to observe the self-focusing is similar to that described in the case of latex spheres suspensions [2]. A $514.5 \mathrm{~nm} \mathrm{cw}$ laser beam is focused by means of a short-focal length microscope objective $(f=10 \mathrm{~mm})$ at the input face of a cell (thickness $1 \mathrm{~mm}$ ) containing the micro-emulsion. The corresponding beam waist is of the order of $3 \mu \mathrm{m}$. The beam propagation in the cell and the intensity profile in the exit plane of the cell are viewed by means of two microscopes (see Fig. 1).

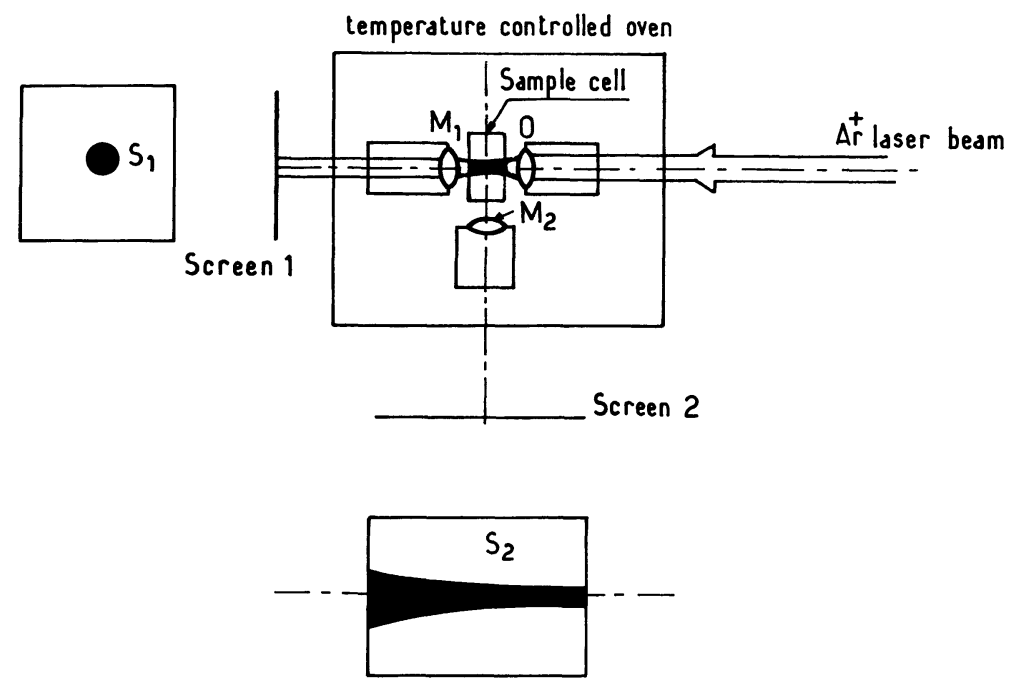

Fig. 1. - Experimental set-up. S1 : transverse section of the beam at the exit plane of the sample cell. S2 : longitudinal section of the beam inside the sample.

The sample used in this study is a four-component micro-emulsion with the following composition in mass percentages : toluene : 72.1 - water : 8.2 - sodium dodecyl sulfate : 3.8 - butanol : 15.9. Toluene has been chosen because of its large index of refraction which leads to a large effective polarizability for the micelles. The phase separation of the system occurs at $T_{\mathrm{c}}=33.2^{\circ} \mathrm{C}$, a temperature at which the correlation length of the fluctuations is estimated to be $\sim 8 \times 10^{-8} \mathrm{~m}$ from Rayleigh light scattering.

In the self-focusing experiments, the micro-emulsion is contained within a cylindrical glass tube (inner diameter $0.5 \mathrm{~mm}$, length $15 \mathrm{~mm}$ ). This tube is located inside the above-mentioned $1 \mathrm{~mm}$ thick glass cell, which is filled with glycerol for ensuring a nearly constant linear index of refraction throughout the cell. The temperature inside the sample is kept constant to within $\pm 0.05^{\circ} \mathrm{C}$.

The onset of self-focusing is manifested by a definite increase of the intensity in the central portion of the beam (Fig. 2c), corresponding to the simultaneous appearance of a bright light filament along the propagation axis (Fig. 2d). For $P>P_{T}$, the intensity profile of the beam is clearly no longer Gaussian (see the rings in Fig. 2c) as a consequence of the spherical aberration of the self-induced lens. In figure 2 the widths of the intense regions are essentially limited by the resolution power of the projection system. No attempt has been made so far to observe the exact structure and diameter of the filament.

The evolution of the threshold power $P_{T}$ as a function of the temperature is shown in figure 3 . Two sets of results from two experiments carried out one month apart from each other have been plotted, showing the reproducibility of the measurements. 


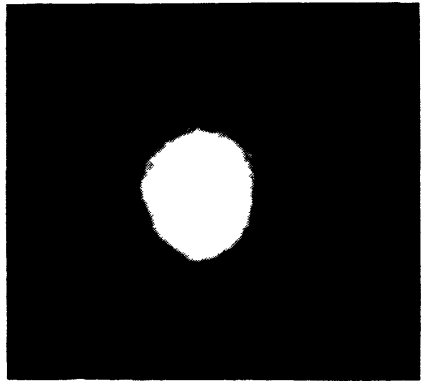

a)

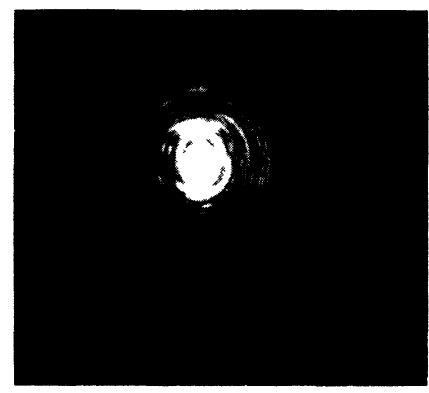

c)

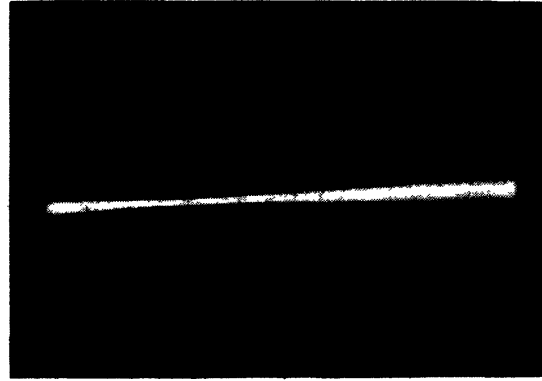

b)

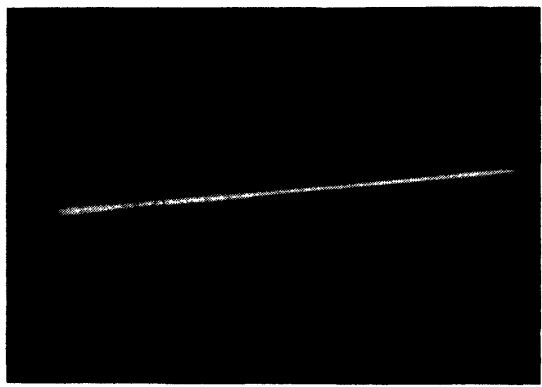

d)

Fig. 2. - Photographs of S1 and S2 (see definitions in the caption of Fig. 1); (a), (b) for a low beam power $\left(P<P_{T}\right)$; (c), (d) for a high beam power $\left(P>P_{T}\right)$.

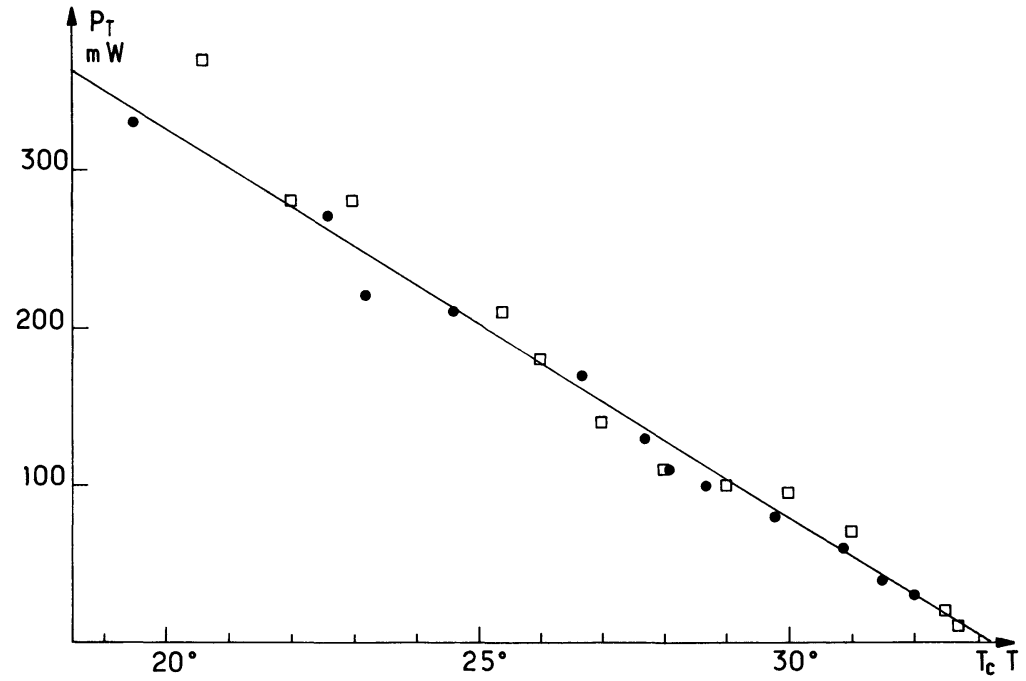

Fig. 3. - Threshold power of self-focusing $P_{T}$ versus temperature. $\bullet, \square$ : data from two sets of experiments. 
As expected $P_{T}$ goes to zero when $T$ gets close to the critical temperature. Powers as small as $10^{-2} \mathrm{~W}$ have been recorded. Fitting the experimental data with equation (9) gives $\gamma=1.0 \pm 0.1$. The time interval needed for the building or the relaxation of a light filament increases from a few seconds at $T_{\mathrm{c}}-T \simeq 10^{\circ} \mathrm{C}$ to a few minutes at $T_{\mathrm{c}}-T \simeq 0.3^{\circ} \mathrm{C}$. This behaviour is in qualitative agreement with the well-known critical slowing down of the density fluctuations near $T_{\mathrm{c}}$. However, because the self-focusing geometry involves a continuous distribution of Fourier modes of the density $N(r)$, and because the structure of the light filament is not known [9], any quantitative analysis of the dynamics of the phenomenon is made very difficult and has not been attempted so far.

The previous results clearly demonstrate that the radiation pressure produces index changes in a micro-emulsion. We have checked that the linear index of refraction of the micro-emulsion is a decreasing function of the temperature. This shows clearly that the laser induced heating of the sample is not responsible for the observed self-focusing. The heating contributes as a diverging lens [11] and slightly modifies the electrostrictive action of the beam because of the non-uniform temperature distribution around the symmetry axis of the set-up. However, the intrinsic absorption of the sample is very small at $541.5 \mathrm{~nm}$ (extinction coefficient $\lesssim 10^{-3} \mathrm{~cm}^{-1}$ ) and as long as convection is absent, a simple calculation shows that the heating of the sample cannot alter the threshold power appreciably [12]. It is nonetheless possible that convective instabilities play an important role in samples with large dimensions. As a matter of fact, the self-focusing could be observed only with samples contained in glass tubes with inner diameters $<1 \mathrm{~mm}$.

Assuming that all the saturation processes of the non-linear index of refraction are negligible [12] at the onset of the self-focusing, $n_{2}$ as given by equation (11) varies from $10^{-9} \mathrm{~cm}^{2} \mathrm{~W}^{-1}$ at $T_{\mathrm{c}}-T$ $=10^{\circ} \mathrm{C}$ to $2.6 \times 10^{-8} \mathrm{~cm}^{2} \mathrm{~W}^{-1}$ at $T_{\mathrm{c}}-T=0.3{ }^{\circ} \mathrm{C}$. The latter value is one order of magnitude larger than that reported for latex spheres suspensions. Another specific advantage of microemulsions in the field of non-linear optical engineering is their stability. Particularly the clumping of solid particles which is frequently observed in the self-trapped light filaments is absent with micro-emulsions.

\section{Conclusion.}

We have demonstrated the capabilities of critical micro-emulsions as a new non-linear material featuring exceptionally high performances.

The very high non-linearities that we have measured are due to the coupling of the electromagnetic field with the number density of micelles near a critical point. Both the amplitude and the dynamics of the response of the system are found to behave critically as a function of the temperature. However the complete quantitative description of the self-focusing of light in a nonlinear medium is still a problem of a considerable difficulty [9] and the results reported here should be considered as semi-quantitative. Particularly it is beyond the scope of this work to provide a conclusive value of the susceptibility exponent $\gamma$. Experiments using a « Forced Rayleigh Scattering " geometry are currently under way and should be much better suited for a quantitative analysis of the critical behaviour of both the statics and the dynamics of the non-linear optical susceptibility.

It should be noted that samples with a high value of $n_{2}$ are also very turbid because $n_{2}$ and the Rayleigh light scattering cross section are proportional quantities. Characterizing such samples by the usual light scattering techniques is made difficult by the related occurrence of multiple scattering of light. In such cases, electrostriction could be a promising alternative.

\section{Acknowledgments.}

We wish to thank D. Roux and A. M. Bellocq for their help and comments and P. Lallemand for helpful remarks concerning the possible role of convective instabilities. 


\section{References}

[1] Palmer, A. J., Opt. Lett. 5 (1980) 54.

[2] Ashrin, A., Dziedzic, J. M., Smith, P. W., Opt. Lett. 7 (1982) 276.

[3] Smith, P. W., Ashrin, A., Tomlinson, W. J., Opt. Lett. 6 (1981) 284.

[4] See for instance Prince, L. M., Micro-emulsions (Academic Press, New York) 1977.

[5] Belloce, A. M., Biais, J., Bothorel, P., Clin, B., Fourche, G., Lalanne, P., Lemaire, B., LemanCEAU, B., Roux, D., Adv. Colloid Interface Sci. 20 (1984) 167.

[6] Huang, J. S., Kim, M. W., Phys. Rev. Lett. 47 (1981) 1462.

Cazabat, A. M., Langevin, D., Meunier, J. and Pouchelon, A., J. Physique Lett. 43 (1982) L-789.

Fourche, G., Belloce, A. M., Brunetti, S., J. Colloid Interface Sci. 88 (1982) 302.

[7] Corti, M., Degiorgio, V., Phys. Rev. Lett. 45 (1980) 1045.

Honorat, Ph., Roux, D., Belloce, A. M., J. Physique, to be published.

Corti, M., Minero, C., Degiorgio, V., J. Phys. Chem. 88 (1984) 309.

[8] Chiao, R. Y., Garmire, E., Townes, C. H., Phys. Rev. Lett. 13 (1968) 479.

[9] Wagner, W. G., Haus, H. A., Marburger, J. H., Phys. Rev. 175 (1968) 256.

[10] Dawnes, E. L., Marburger, J. H., Phys. Rev. 179 (1969) 862.

[11] Akhmanov, S. A., Khokhlov, R. V., Sukhorukov, A. P., in Laser Handbook, F. T. Arecchi and E. O. Schulz-Dubois, eds (North-Holland, Amsterdam) 1972, vol. 2, part E2.

[12] AfrFI, M., Thesis, Bordeaux (1984) unpublished. 